Check for updates

Cite this: Mater. Chem. Front. 2019, 3, 916

Received 30th January 2019, Accepted 25th March 2019

DOI: 10.1039/c9qm00059c

rsc.li/frontiers-materials

\section{Supramolecular nanocatalyst in water: successive click-driven assembly of click-derived rod amphiphiles $\dagger$}

\author{
Inhye Kim (D) ab and Eunji Lee (D) *a
}

A supramolecular vesicular nanocatalyst (VC) for successive Cu'-catalyzed azide-alkyne cycloaddition in water was developed by self-assembly of a click chemistry-derived rod amphiphile (CA). The CA generated by click reaction of azide-terminated benzyl ether dendrons and diethynyl naphthalene selfassembles into vesicles in water. The excellent catalytic activity of the VC was endowed by $\mathrm{Cu}^{\prime}$-chelation to triazole-containing rod block within VC wall. The VC performed click reaction of benzyl azide with phenylacetylene or trimethylsilylacetylene. The resultant hydrophobic products self-encapsulated in VC wall affect the interfacial curvature of the VC according to their structural compatibility with CA, inducing the increase in vesicular size or structural change to micelle. Interestingly, the morphology of VC-derived nanocatalyst was further controlled from micelle to vesicle and vice versa by successive click reactions, leading to controllable loading/release of hydrophilic payloads. This recyclable catalytic activity of the selftransformable nanocatalyst was confirmed by a visually detectable click reaction offering a fluorescent color change of the aqueous VC solution.

\section{Introduction}

Supramolecular nanovesicles based on the aqueous selfassembly of amphiphiles possess two types of confined spaces: the aqueous core cavity and the hydrophobic wall. ${ }^{1-3}$ The confined wall enables the vesicle to serve as a nanoreactor by providing a hydrophobic environment, where chemical reactions such as a Diels-Alder reaction, ${ }^{4}$ palladium-catalyzed carboncarbon bond forming reaction, ${ }^{5,6}$ and enzymatic cascade reaction $^{7-9}$ can be performed. This ability can be used to increase the conversion efficiency by locally concentrating the reacting species and pre-organizing them in a favored conformation for a desired reaction. ${ }^{10}$ The vesicles can also show a nanostructural change in response to external stimuli such as pH, light, temperature, and guest molecules, ${ }^{11-13}$ resulting from the interfacial curvature change of vesicles by the dynamic assembly behavior of stimuli-responsive block-containing amphiphiles. ${ }^{14,15}$ This makes them attractive candidates for developing transformable nanomaterials in smart chemo/biosensors and controlled drug/imaging agent delivery. ${ }^{14-17}$ However, self-transformable

\footnotetext{
${ }^{a}$ School of Materials Science and Engineering, Gwangju Institute of Science and Technology, 123 Cheomdangwagi-ro, Buk-gu, Gwangju 61005, Republic of Korea. E-mail: eunjilee@gist.ac.kr

${ }^{b}$ Graduate School of Analytical Science and Technology, Chungnam National University, 99 Daehak-ro, Yuseong-gu, Daejeon 34134, Republic of Korea $\dagger$ Electronic supplementary information (ESI) available. See DOI: 10.1039/ c9qm00059c
}

nanoreactors that can act as an on-demand nanotransporter of payloads has rarely been reported.

$\mathrm{Cu}^{\mathrm{I}}$-catalyzed azide-alkyne cycloaddition (CuAAC) click chemistry, resulting in 1,4-disubstituted 1,2,3-triazole derivatives, ${ }^{18-20}$ has led to a wealth of applications due to its synthetic simplicity, mild reaction conditions, and biocompatibility. ${ }^{21-24}$ However, the instability of catalytically active $\mathrm{Cu}^{\mathrm{I}}$ sites requires a large amount of $\mathrm{Cu}^{\mathrm{I}}$ catalyst to accelerate the CuAAC click reaction. The complete removal of $\mathrm{Cu}^{\mathrm{I}}$ is also a challenge and limits the utilization of click chemistry in electronics, biomedicine, and green chemistry. ${ }^{25}$ Interestingly, the clickderived triazole group has also been attractive as a ligand providing several donor sites for chelating metal ions, ${ }^{26,27}$ increasing its use in click chemistry with applications such as chemosensing, ${ }^{28}$ catalysis, ${ }^{29}$ and therapeutics. ${ }^{30,31}$ Therefore, one can envision that the click reaction-derived rod amphiphiles spontaneously form vesicles in water, providing a hydrophobic space where catalytic $\mathrm{Cu}^{\mathrm{I}}$ is chelated by a triazole group and can act as a vesicular nanocatalyst (VC) for click reactions.

Herein, we report the supramolecular self-transformable VC based on the self-assembly of a click-derived, $\mathrm{Cu}^{\mathrm{I}}$-chelating rod amphiphile (Fig. 1). The VC can carry out successive click reactions with an exceptional conversion efficiency owing to (i) stabilized catalytic $\mathrm{Cu}^{\mathrm{I}}$ and reactants preserving the catalytic activity, and (ii) the reactive species in close proximity to the catalytic sites in the VC wall. Also, the self-encapsulation of the hydrophobic click products within the wall affects the 


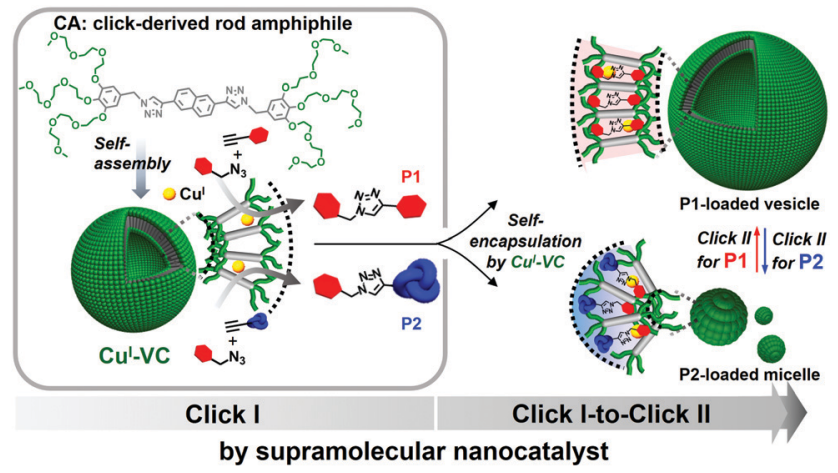

Fig. 1 Interfacial curvature-controllable supramolecular vesicular nanocatalyst (VC) for successive click I-to-click II reactions, formed by selfassembly of a click-derived rod amphiphile (CA) in water, depending on the molecular structure similarity of click reaction products $\mathbf{P} \mathbf{1}$ and $\mathbf{P} \mathbf{2}$ with the rod segment of CA. P1 and $\mathbf{P} \mathbf{2}$ are the click reaction products of benzyl azide with phenylacetylene or trimethylsilylacetylene, respectively.

interfacial curvature and thus the morphology of the VC, enabling the extensive use of VCs as smart nanomaterials.

\section{Results and discussion}

The click-derived rod amphiphile (CA) was synthesized by the CuAAC reaction of azide-terminated benzyl ether dendrons and diethynyl naphthalene (Fig. S1-S3, ESI $\dagger$ ) and showed vesicular formation by aqueous self-assembly (Fig. 1 and Fig. S6b, ESI $\dagger$ ). The $\mathrm{Cu}^{\mathrm{II}}$-chelation to the triazolyl moiety of $\mathbf{C A}$, to provide the $\mathrm{Cu}^{\mathrm{I}}$-chelated $\mathbf{V C}\left(\mathbf{C} \mathbf{u}^{\mathrm{I}}-\mathbf{V C}\right)$, was examined by ultraviolet-visible (UV-Vis), fluorescence, and ${ }^{1} \mathrm{H}$ nuclear magnetic resonance (NMR) spectroscopies (Fig. S6 and S7, $\mathrm{ESI}_{\dagger}$ ). $\mathrm{CuSO}_{4}$ (0.5 equiv. mol to $\mathbf{C A}$ ) and sodium ascorbate (NaAsc, 3.0 equiv. mol to $\mathrm{CuSO}_{4}$ ) were added to an aqueous solution of $\mathbf{C A}$ for the in situ reduction of $\mathrm{Cu}^{\mathrm{II}}$ to $\mathrm{Cu}^{\mathrm{I}}$ (Fig. 2). The stoichiometric ratio of $\mathrm{CA}: \mathrm{Cu}^{\mathrm{II}}$ was $2: 1$ (Fig. S6c, ESI $\dagger$ ). ${ }^{32}$ In the UV-Vis spectra, the characteristic d-d transition of $\mathrm{Cu}^{\mathrm{II}}$ in the range $700-900 \mathrm{~nm}^{33}$ disappeared, and new absorption peaks appeared at 600 and $750 \mathrm{~nm}$ after addition of $\mathrm{CuSO}_{4}$ to the aqueous solution of $\mathbf{C A}$, which could be assigned to a metal-to-ligand-transfer transition (Fig. S6d, ESI $\dagger$ ). ${ }^{34}$ The two peaks at 600 and $750 \mathrm{~nm}$ disappeared, and a band at $670 \mathrm{~nm}$ appeared after further reduction of $\mathrm{Cu}^{\mathrm{II}}$ to $\mathrm{Cu}^{\mathrm{I}}$ by NaAsc, indicating $\mathrm{Cu}^{\mathrm{I}}$-chelation by the triazolyl group of CA. The decrease in emission of $\mathbf{C A}$ after addition of $\mathrm{CuSO}_{4}$, indicative of the $\mathrm{Cu}^{\mathrm{II}}$-chelation by the triazole group of $\mathbf{C A}$, could be attributed to the reverse photoinduced electron transfer ${ }^{35}$ involving electron donation from the excited naphthalene unit to the $\mathrm{Cu}^{\mathrm{II}}$-binding triazole group (Fig. S6e, ESI $\dagger$ ). ${ }^{36} \mathrm{The}^{1} \mathrm{H}$ NMR signal of the triazole proton $\left(\mathrm{H}_{\mathrm{c}}\right)$ of $\mathbf{C A}$ at $8.16 \mathrm{ppm}$ measured in $\mathrm{D}_{2} \mathrm{O}$
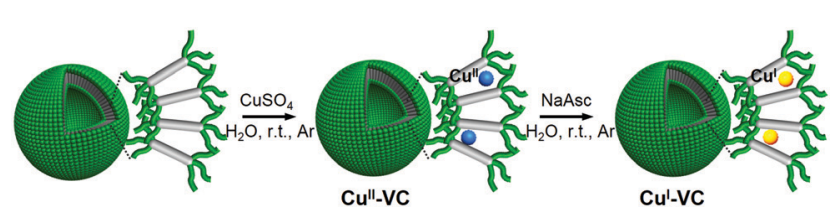

Fig. 2 Development of the Cu'-vC for CuAAC. shifted upfield to 8.12 ppm upon addition of $\mathrm{CuSO}_{4}$ (Fig. S7a, ESI $\dagger$ ). ${ }^{25}$ The $\mathrm{H}_{\mathrm{g}}$ of $\mathbf{C A}$ shifted upfield by $0.06 \mathrm{ppm}$, suggesting the oxygen atoms next to the phenyl group were interacting with $\mathrm{Cu}^{\text {II }}$. After further addition of NaAsc, the $\mathrm{H}_{\mathrm{c}^{-}}$and $\mathrm{H}_{\mathrm{g}}$-signals showed a downfield shift of 0.04 and $0.03 \mathrm{ppm}$, respectively, indicating $\mathbf{C A}-\mathrm{Cu}^{\mathrm{I}}$ complex formation. Fig. S7b (ESI $\dagger$ ) shows the plausible binding model of $\mathbf{C A}-\mathrm{Cu}^{\mathrm{I}}$. The successful fabrication of vesicular structures of $\mathbf{C A}-\mathrm{Cu}^{\mathrm{I}}$ for $\mathbf{C} \mathbf{u}^{\mathrm{I}}-\mathbf{V C}$ was confirmed by transmission electron microscopy (TEM) (Fig. S8, ESI $\dagger$ ). The unstained TEM images of both vesicular $\mathbf{C A}-\mathrm{Cu}^{\mathrm{II}}$ precatalyst and $\mathbf{C A}-\mathrm{Cu}^{\mathrm{I}}$ catalyst clearly showed the vesicular wall with enhanced contrast due to the chelation of $\mathrm{Cu}$ by the triazole group at the hydrophobic segment.

The catalytic ability of $\mathbf{C u}^{\mathbf{I}}$-VC was assessed by a ${ }^{1} \mathrm{H}$ NMR study. First, the CuAAC reaction using water-insoluble benzyl azide (5 mol equiv. to $\mathbf{C A}$ for $\mathbf{C u}^{\mathrm{I}}-\mathbf{V C}$ ) and phenylacetylene (5 equiv.) was performed for $24 \mathrm{~h}$ to generate click product P1 in the presence of $\mathbf{C u}^{\mathrm{I}}-\mathbf{V C}$ (Fig. S5a, ESI $\dagger$ and Table 1). After dissolving the resultant nanocatalyst in $\mathrm{CDCl}_{3}$, a conversion efficiency of $99 \%$ was reached (Table 1 , entry 1 ). The reaction did not occur in the absence of $\mathbf{C u}^{\mathbf{I}}-\mathbf{V C}$ (entry 2). The enhanced reactivity of $\mathbf{C u}^{\mathrm{I}}-\mathbf{V C}$ was confirmed by comparison to the reaction with $\mathrm{Cu}^{\mathrm{I}}$, showing moderate catalytic conversion of $31 \%$ (entry 3). This could be attributed to a closer contact of preorganized hydrophobic reactants to stabilized catalytically active $\mathrm{Cu}^{\mathrm{I}}$ sites confined in the walls of the supramolecular $\mathbf{C} \mathbf{u}^{\mathrm{I}}-\mathbf{V C}{ }^{37}$

Consequently, the water-insoluble click product $\mathbf{P 1}$ was generated and self-encapsulated in the hydrophobic vesicular membrane. The ${ }^{1} \mathrm{H}$ NMR spectrum of P1-loaded $\mathbf{C u}^{\mathbf{I}}-\mathbf{V C}$ in $\mathrm{D}_{2} \mathrm{O}$ showed the upfield shift of naphthyl protons $\left(\mathrm{H}_{\mathrm{d}}, \mathrm{H}_{\mathrm{e}}\right.$, and $\mathrm{H}_{\mathrm{f}}$, around $0.10-0.14 \mathrm{ppm})$, benzylic $\mathrm{H}_{\mathrm{b}}(0.05 \mathrm{ppm})$, and triazolyl $\mathrm{H}_{\mathbf{c}}(0.06 \mathrm{ppm})$ protons of $\mathbf{C u}^{\mathrm{I}}-\mathbf{V C}$ compared with that of the original one in $\mathrm{D}_{2} \mathrm{O}$ (Fig. S9c, ESI $\dagger$ ). The upfield shifts of $\mathrm{H}_{\mathrm{b}}, \mathrm{H}_{c}$,

Table 1 Comparison of VC catalyst efficiency in CuAAC ${ }^{a}$

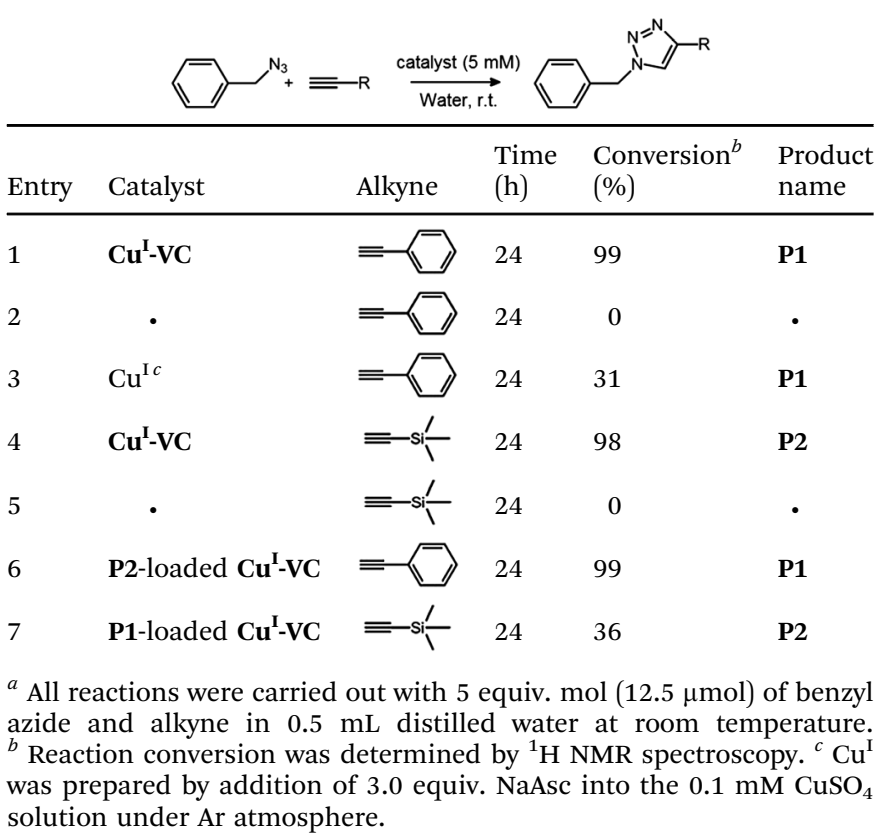



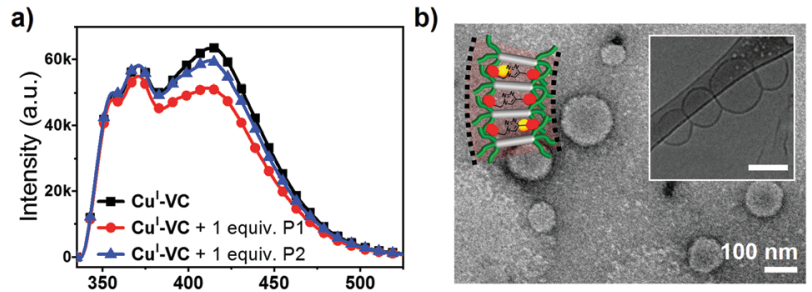

c)
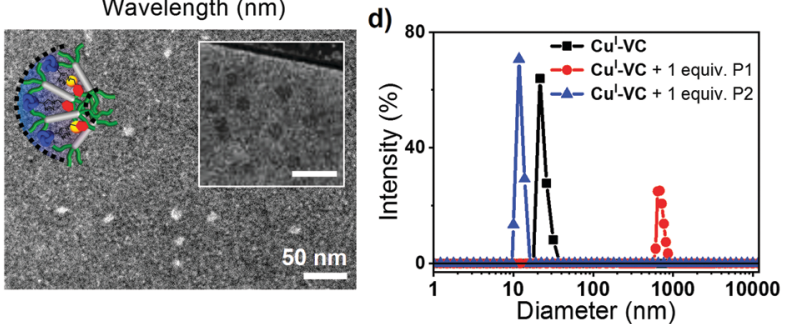

Fig. 3 (a) Emission spectra ( $\lambda_{\mathrm{ex}}=260 \mathrm{~nm}$ ) of Cu'-VC, P1-, and P2-loaded Cu'-VC in water. Negatively stained TEM image of (b) P1- and (c) P2-loaded Cu'-vC (0.02 mM) with 2 wt\% uranyl acetate. Insets of (b and c) show the cryo-TEM image. Inset scale bar equals (b) 100 and (c) $50 \mathrm{~nm}$, respectively. (d) Hydrodynamic diameters of Cu'-VC, P1-, and P2-loaded Cu'-VC

$\mathrm{H}_{\mathrm{d}}, \mathrm{H}_{\mathrm{e}}$, and $\mathrm{H}_{\mathrm{f}}$ could be ascribed to the shield effect of P1 by the $\pi-\pi$ stacking with the rod amphiphile CA. ${ }^{38}$ The absorbance of $\mathbf{C u}$-VC decreased and the emission was quenched after generation of P1 by the CuAAC reaction (Fig. S10, ESI $\dagger$ and Fig. 3a)

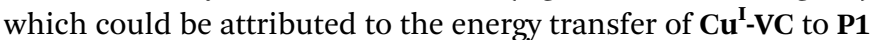
due to the $\pi-\pi$ stacking between the aromatic block of CA and P1. ${ }^{39}$ TEM images revealed that the vesicular structure of $\mathbf{C} \mathbf{u}^{\mathbf{I}}-\mathbf{V C}$ remains intact after loading $\mathbf{P 1}$ by the CuAAC reaction (Fig. 3b). Even in the presence of excess reactants, precipitating P1 (white solid) by the constant reactivity of $\mathbf{C u}^{\mathbf{I}}-\mathbf{V C}$, the vesicular structure was maintained without rupture (Fig. S11a and b, ESI $\dagger$ ).

To confirm the catalytic activity of $\mathbf{C} \mathbf{u}^{\mathbf{I}}-\mathbf{V C}, \mathbf{P} 2$ with a bulky trimethyl group was synthesized using the CuAAC reaction of trimethylsilylacetylene and benzyl azide (Fig. 1, Table 1 and Fig. S5b, ESI $\dagger$ ). Cu $\mathbf{C u}^{\mathbf{I}}-\mathbf{V C}$ shows an excellent conversion efficiency of $98 \%$ (entry 4). When the P2-forming CuAAC reaction was carried out without $\mathbf{C u}^{\mathbf{I}}-\mathbf{V C}$, the reaction did not occur as expected (entry 5). The ${ }^{1} \mathrm{H}$ NMR spectrum of $\mathbf{C} \mathbf{u}^{\mathbf{I}}-\mathbf{V C}$ with $\mathbf{P 2}$ measured in $\mathrm{D}_{2} \mathrm{O}$ showed an upfield shift similar to that observed for P1-loaded $\mathbf{C u}^{\mathbf{I}}$-VC in $\mathrm{D}_{2} \mathrm{O}$, indicating the encapsulation of $\mathbf{P 2}$ by $\mathbf{C u}^{\mathbf{I}}-\mathbf{V C}$ (Fig. S12c, ESI $\dagger$ ). The P2-loaded $\mathbf{C u}^{\mathbf{I}}-\mathbf{V C}$ showed the formation of micelles of diameter $\sim 10 \mathrm{~nm}$ (Fig. 3c and Fig. S13c, ESI $\dagger$ ), which is twice the molecular length of fully extended CA (Fig. S6a, ESI $\dagger$ ). The emission quenching of $\mathbf{C u}^{\mathbf{I}}-\mathbf{V C}$ upon generation of $\mathbf{P 2}$ suggests the intercalation of $\mathbf{P 2}$ within the hydrophobic wall of $\mathbf{C u}^{\mathbf{I}}-\mathbf{V C}$ (Fig. 3a). The smaller decrease in the emission of P2-loaded $\mathbf{C} \mathbf{u}^{\mathbf{I}}$-VC than that of P1-loaded $\mathbf{C} \mathbf{u}^{\mathbf{I}}-\mathbf{V C}$ could be the result of less effective $\pi-\pi$ stacking of $\mathbf{P 2}$ with the hydrophobic rod segments of the CA nanocatalyst. ${ }^{40}$ The bulky trimethyl groups of $\mathbf{P 2}$ may hinder the close packing of CA within the membrane of $\mathbf{C u}^{\mathbf{I}}$-VC, resulting in morphological transformation of $\mathbf{C} \mathbf{u}^{\mathbf{I}}$-VC from vesicle to micelle. Dynamic light scattering (DLS) revealed an increase in the average hydrodynamic diameter $\left(R_{\mathbf{H}}\right)$ of P1-intercalated $\mathbf{C u}^{\mathbf{I}}-\mathbf{V C}$ (from $\sim 21 \mathrm{~nm}$ to $\sim 600 \mathrm{~nm}$ ) (Fig. $3 \mathrm{~d}$ ). A decrease in the $R_{\mathrm{H}}$ of P2-intercalated $\mathbf{C} \mathbf{u}^{\mathbf{I}}-\mathbf{V C}$ was observed (from $\sim 21 \mathrm{~nm}$ to $\sim 10 \mathrm{~nm}$ ) compared with $\mathbf{C} \mathbf{u}^{\mathbf{I}}$-VC. Therefore, the molecular structure similarity of the click reaction products with the hydrophobic rod segment of CA may affect the interfacial curvature of supramolecular nanocatalyst $\mathbf{C u}^{\mathbf{I}}-\mathbf{V C}$, resulting in a morphological change. For instance, the high structural compatibility of $\mathbf{P 1}$ with $\mathbf{C A}$ could enable the effective encapsulation of $\mathbf{P 1}$ within the membrane of $\mathbf{C u}^{\mathbf{I}}-\mathbf{V C}$. The $\pi-\pi$ stacking of P1 with CA could be optimized as $\mathbf{P 1}$ and the rigid hydrophobic segment of CA are arranged in parallel, which decreased the interfacial curvature of the wall, leading to an increase in Cu'-VC size. ${ }^{41}$ These results are consistent with the entropic cost of intercalating molecules with increased volume, which is responsible for the vesicle-to-micelle morphological change. ${ }^{42}$

As the interfacial curvature of $\mathbf{C} \mathbf{u}^{\mathbf{I}}-\mathbf{V C}$ can be tuned by the molecular architecture of click products, we studied whether the reversible morphological transformation of nanocatalyst from micellar aggregates to vesicles could be achieved by sequential click reactions to form P1 using P2-loaded $\mathbf{C} \mathbf{u}^{\mathbf{I}}$-VC (Fig. 4a). The CuAAC reaction of benzyl azide and phenylacetylene catalyzed by P2-loaded $\mathbf{C u}^{\mathbf{I}}$-VC was performed (Table 1, entry 6). The catalytic activity of the P2-loaded nanoreactor still shows a tremendous conversion efficiency of $99 \%$ despite the pre-loading of $\mathbf{P 2}$ in $\mathbf{C} \mathbf{u}^{\mathbf{I}}$-VC. In the ${ }^{1} \mathrm{H}$ NMR spectrum of P2-loaded $\mathbf{C u}^{\mathbf{I}}-\mathbf{V C}$ after the CuAAC reaction to form $\mathbf{P 1}$ in $\mathrm{D}_{2} \mathrm{O}, \mathrm{H}_{\mathrm{b}}, \mathrm{H}_{\mathrm{c}}, \mathrm{H}_{\mathrm{d}}, \mathrm{H}_{\mathrm{e}}$, and $\mathrm{H}_{\mathrm{f}}$ are significantly downfield shifted (0.05-0.09 ppm), probably indicating a strong hydrogen bonding interaction between the triazolyl and naphthyl protons of CA and the aromatic protons and nitrogen atoms in the triazole moiety of P1 and P2 (Fig. 4b). ${ }^{38}$ $\mathrm{H}_{\mathrm{a}}$ and $\mathrm{H}_{\mathrm{g}}$ showed a moderate downfield shift (0.05 ppm), which suggests the enhanced hydrogen bonding interaction of polyethylene oxide chains of CA with water. However, after dissolving the resultant nanocatalyst in $\mathrm{CDCl}_{3}$, only $\mathbf{P 1}$ signals were detected in the ${ }^{1} \mathrm{H}$ NMR spectrum (Fig. $4 \mathrm{~b}$ and Fig. S14, ESI $\dagger$ ). Fluorescence quenching of P2-loaded $\mathbf{C u}^{\mathbf{I}}$-VC was observed after the P1-forming CuAAC reaction (Fig. S15b, ESI $\dagger$ ). The TEM experiment showed the presence of vesicles (Fig. 4c). These results can be explained by preloaded $\mathbf{P} 2$ in $\mathbf{C} \mathbf{u}^{\mathbf{I}}-\mathbf{V C}$ being replaced with $\mathbf{P 1}$ and released into the aqueous solution because P1 has greater molecular structure compatibility with CA, which can form a more stable vesicle through $\pi$-stacking. The released $\mathbf{P 2}$ to the aqueous phase was confirmed by ${ }^{1} \mathrm{H}$ NMR experiment. The transformation of P2-loaded nanocatalyst to a vesicular structure by successive click reactions was further confirmed using hydrophilic dye fluorescein isothiocyanate (FITC) that can be encapsulated in the interior cavity of the vesicle (Fig. S16a, ESI $\dagger$ ). ${ }^{43}$ As expected, the fluorescence micrograph clearly showed bright green-emitting spherical aggregates, indicating the formation of a vesicle (Fig. 4d and Fig. S16b, ESI $\dagger$ ).

The morphological transformation of P1-loaded nanocatalyst was also demonstrated by conducting P2-forming CuAAC reaction (Fig. 5, Fig. S14 and S17, ESI $\dagger$ ). The P1-loaded vesicular nanocatalyst encapsulating FITC in inner aqueous compartment could release the FITC fluorescent dyes into the outer aqueous phase after performing a P2-forming CuAAC reaction as a result 
a)

b)
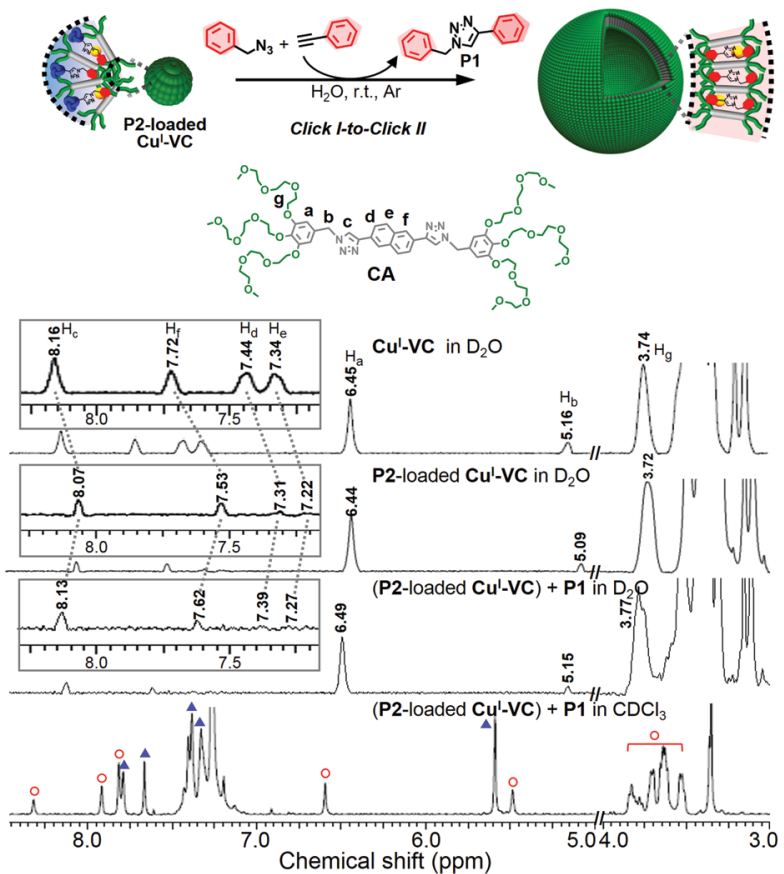

c)

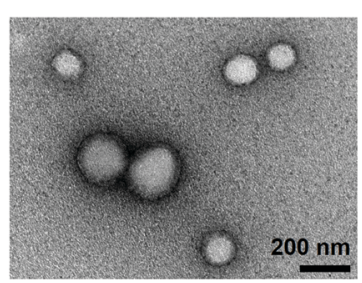

d)

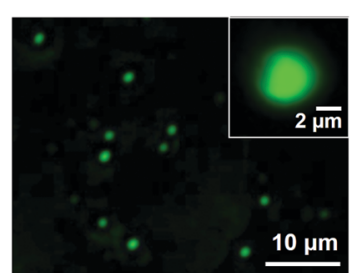

Fig. 4 (a) Successive click reactions of nanocatalyst, resulting in the morphological change of $\mathrm{Cu}^{\prime}$-VC from micelle to vesicle. (b) ${ }^{1} \mathrm{H}$ NMR spectra of $\mathbf{C} \mathbf{u}^{\prime}-\mathbf{V C}$ and $\mathbf{P} \mathbf{2}$-loaded $\mathbf{C u}^{\mathbf{l}}$-VC measured in $\mathrm{D}_{2} \mathrm{O}$ and $\mathbf{P} \mathbf{2}$-loaded $\mathrm{Cu}^{\prime}$-VC after click reaction to generate $\mathbf{P} \mathbf{1}$ measured in $\mathrm{D}_{2} \mathrm{O}$ and $\mathrm{CDCl}_{3}$. Red hollow circle $(O)$ and blue triangle $(\boldsymbol{\Delta})$ denote $C A$ and $\mathbf{P} \mathbf{1}$, respectively. More detailed information is in the ESI. $\dagger$ (c) Negatively stained TEM image and (d) fluorescence micrograph of the P2-loaded C $\mathbf{u}^{\prime}$-VC after click reaction to synthesize $\mathbf{P} \mathbf{1}$. Inset of (d) shows the vesicle containing FITC within the inner aqueous medium separated by a wall from the outer aqueous solution. Green fluorescence: FITC.

of the nanostructural transformation to micelle (Fig. 5b). The TEM image clearly showed the micellar structures with a diameter of $\sim 10 \mathrm{~nm}$ (Fig. $5 \mathrm{c}$ ). The ${ }^{1} \mathrm{H}$ NMR spectrum of the resultant micelles dissolved in $\mathrm{CDCl}_{3}$ indicated the presence of both P1 and P2 within the micelle core (Fig. S14, ESI $\dagger$ ) which could be because the preloaded $\mathbf{P 1}$ is not fully replaced with $\mathbf{P 2}$ due to the higher structural compatibility of $\mathbf{P 1}$ with CA. The conversion efficiency of $\mathbf{P} 2$ production using P1-loaded $\mathbf{C} \mathbf{u}^{\mathbf{I}}-\mathbf{V C}$ was calculated to be relatively low (36\%) (Table 1, entry 7 ). This result could be attributed to the strong mutual interaction between CA and P1, limiting the access of trimethylsilylacetylene and benzyl azide to the catalytic $\mathrm{Cu}^{\mathrm{I}}$ located in the vesicular wall. Therefore, the molecular packing constraint induced by preloaded products within the hydrophobic wall of the nanocatalyst should be considered for performing successive click reactions.

The recyclable catalytic activity of $\mathbf{C u}^{\mathbf{I}}-\mathbf{V C}$ with structural transformable capability was further confirmed by conducting

a)

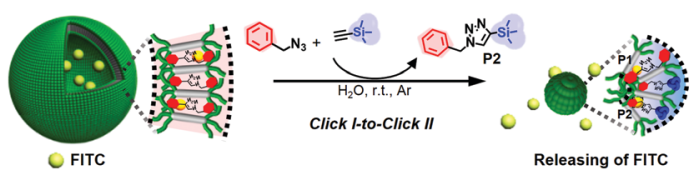

b)

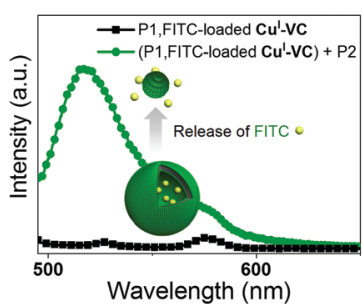

c)

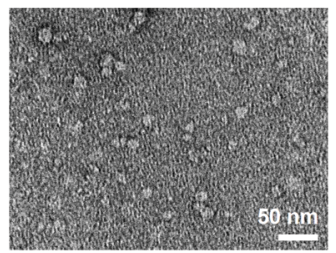

Fig. 5 (a) Morphological transformation of $\mathbf{C u}^{\prime}-\mathbf{V C}$ from vesicle to micelle by successive click reactions of nanocatalyst, leading to controllable loading/release of FITC payloads. (b) Emission enhancement of the FITC-released aqueous solution of P1-loaded Cu'-VC $(0.5 \mathrm{mM})$ incorporating FITC after click reaction to form P2. (c) Negatively stained TEM image of P1-loaded $\mathbf{C u}^{\prime}$-VC after $\mathbf{P} 2$-forming CuAAC reaction with 2 wt\% uranyl acetate.

a visually detectable click reaction via a fluorescent color change of a $\mathbf{C} \mathbf{u}^{\mathbf{I}}-\mathbf{V C}$ solution. The conversion of water-insoluble nonfluorescent reactants to fluorescent products by the CuAAC reaction can help detect the click reaction using the naked eye. The hydrophobic coumarin derivatives were utilized as a target click product. Upon adding coumarin into aqueous $\mathbf{C u}^{\mathbf{I}}-\mathbf{V C}$ solution, characteristic absorption of coumarin $(\sim 280 \mathrm{~nm})$ appeared while the fluorescence of $\mathbf{C} \mathbf{u}^{\mathbf{I}}-\mathbf{V C}$ was quenched, indicating the presence of coumarin within the hydrophobic segment of $\mathbf{C u}^{\mathrm{I}}$-VC (Fig. S18, ESI $\dagger$ ). To generate a coumarin-based fluorescent click product with different structural similarities to CA, we synthesized 3-azido-7-hydroxycoumarin, a nonfluorescent azide (Fig. S4, ESI $\dagger$ ). ${ }^{4,45}$ The fluorescent emission of the coumarin moiety in 3-azido-7-hydroxycoumarin is quenched by the lone pair of electrons from the azido group. ${ }^{46,47}$ Phenylacetylene and trimethylsilylacetylene were also used for the CuAAC reactions with 3-azido-7-hydroxycoumarin (Fig. 6a). The resulting click products of phenylacetylene and trimethylsilylacetylene with 3-azido-7-hydroxycoumarin were referred to as CP1 and CP2, respectively (Fig. 6a). As expected, the blue color of $5 \mathrm{mM}$ Cu'-VC solution became turquoise under irradiation at $365 \mathrm{~nm}$, indicating the formation of CP1 and the subsequent loading within the $\mathbf{C} \mathbf{u}^{\mathbf{I}}-\mathbf{V C}$ vesicular membrane. In contrast, the CP2-containing $\mathbf{C} \mathbf{u}^{\mathbf{I}}$-VC solution showed relatively weak green emission under UV irradiation. A new absorption band around $340 \mathrm{~nm}$ appeared for both CP1- and CP2-loaded $\mathbf{C u}^{\mathbf{I}}$-VC (Fig. 6b), and the fluorescence of $\mathbf{C u}^{\mathbf{I}}-\mathbf{V C}$ was quenched (Fig. 6c). These results indicate that click products $\mathbf{C P 1}$ and $\mathbf{C P 2}$ were incorporated within the $\mathbf{C} \mathbf{u}^{\mathbf{I}}-\mathbf{V C}$ wall. Notably, a characteristic emission peak of CP1 at $480 \mathrm{~nm}$ appeared for CP1-loaded $\mathbf{C u}^{\mathbf{I}}-\mathbf{V C}$ when excited at the wavelength of $260 \mathrm{~nm}$ which indicates the fluorescence resonance energy transfer (FRET) due to the close proximity of CP1 and the rod block of CA. ${ }^{48}$ The electron lone pair of the azido moiety of 3-azido-7-hydroxycoumarin could be localized as the triazole ring is formed in $\mathbf{C P 1}$, leading to the activation of 
a)

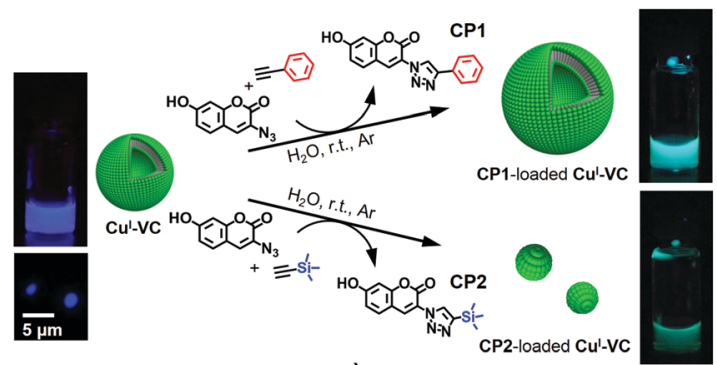

b)

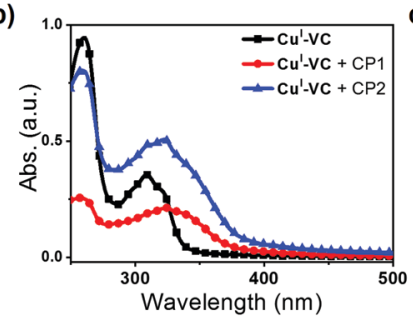

d)

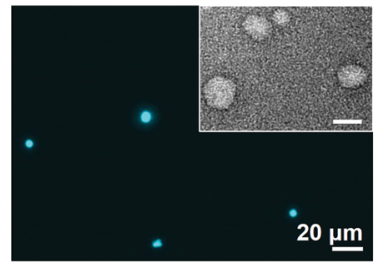

c)
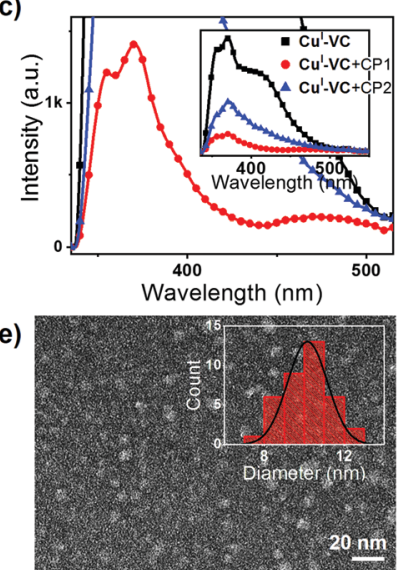

Fig. 6 (a) $\mathrm{Cu}^{\prime}-\mathrm{VC}$ nanocatalyst with images of a $5 \mathrm{mM} \mathrm{Cu}$ '-VC solution under UV irradiation $\left(\lambda_{\mathrm{ex}}=365 \mathrm{~nm}\right)$. Change in (b) absorption and (c) emission spectra of $0.02 \mathrm{mM} \mathrm{Cu}$-VC upon CuAAC reaction to generate CP1 and CP2, respectively $\left(\lambda_{\mathrm{ex}}=260 \mathrm{~nm}\right)$. (d) Fluorescence micrograph of CP1-loaded Cu'-VC. Inset shows the negatively stained TEM image of CP1-loaded Cu'-VC with a scale bar of $50 \mathrm{~nm}$. (e) Negatively stained TEM image of CP2-incorporated $\mathbf{C u}^{\prime}$-VC. Inset shows the diameter distribution of the micelle.

fluorescence. $^{46,47}$ The emergence of the FRET signal demonstrated efficient stacking between CP1 and the rod amphiphile CA. The fluorescence micrograph of the CP1-loaded $\mathbf{C u}^{\mathbf{I}}-\mathbf{V C}$ confirmed the presence of spherical structures (Fig. 6d), and TEM revealed the intact vesicular structure of CP1-encapsulated $\mathbf{C} \mathbf{u}^{\mathbf{I}}-\mathbf{V C}$ (inset of Fig. 6d and Fig. S19, ESI $\dagger$ ). Loading of CP2 with an unsymmetric structure imposed by the bulky trimethyl group into $\mathbf{C u}^{\text {I}}$-VC showed the formation of the micellar aggregates (Fig. 6e). These results are consistent with the morphological transition from vesicles to micelles observed for $\mathbf{C u}^{\mathbf{I}}$-VC when synthesizing $\mathbf{P 1}$ and $\mathbf{P 2}$ based on the benzyl azide.

\section{Conclusions}

In summary, we have developed an aqueous supramolecular nanocatalyst based on the self-assembly of the click-derived rod amphiphile, capable of successive click reactions, which maintained excellent catalytic efficiency. The self-transformable behavior of the nanocatalyst was controlled by encapsulating structurally compatible hydrophobic click reaction products with a rod amphiphile, which, in turn, affects the interfacial curvature of the nanocatalyst. The morphological transformability enables the click nanoreactor/catalyst potential for in situ production of on demand payloads and controllable loading/release of such payloads, including drugs, imaging agents, and chemical species. This can further broaden the potential applications of supramolecular nanocatalysts into the field of smart nanobiomedicine and chemo/biosensors.

\section{Conflicts of interest}

There are no conflicts of interest to declare.

\section{Acknowledgements}

This research was supported by Basic Science Research Program (2016R1A2B4012322) through the National Research Foundation of Korea (NRF). We thank Prof. B. K. Cho for the discussion of amphiphile CA synthesis.

\section{References}

1 P. Tanner, P. Baumann, R. Enea, O. Onaca, C. Palivan and W. Meier, Acc. Chem. Res., 2011, 44, 1039-1049.

2 C. LoPresti, H. Lomas, M. Massignani, T. Smart and G. Battaglia, J. Mater. Chem., 2009, 19, 3576-3590.

3 D. E. Discher and A. Eisenberg, Science, 2002, 297, 967-973.

4 T. Rispens and J. B. F. N. Engberts, Org. Lett., 2001, 3, 941-943.

5 G. Hamasaka, T. Muto and Y. Uozumi, Angew. Chem., Int. Ed., 2011, 50, 4876-4878.

6 M. Liu, X. Zhu, L. Wu, X. Zhou, J. Li and J. Ma, RSC Adv., 2015, 5, 38264-38270.

7 R. J. R. W. Peters, I. Louzao and J. C. M. van Hest, Chem. Sci., 2012, 3, 335-342.

8 D. M. Vriezema, P. M. L. Garcia, N. S. Oltra, N. S. Hatzakis, S. M. Kuiper, R. J. M. Nolte, A. E. Rowan and J. C. M. van Hest, Angew. Chem., Int. Ed., 2007, 46, 7378-7382.

9 O. Rifaie-Graham, S. Ulrich, N. F. B. Galensowske, S. Balog, M. Chami, D. Rentsch, J. R. Hemmer, J. R. de Alaniz, L. F. Boesel and N. Bruns, J. Am. Chem. Soc., 2018, 140, 8027-8036.

10 D. M. Vriezema, M. C. Aragonès, J. A. A. W. Elemans, J. J. L. M. Cornelissen, A. E. Rowan and R. J. M. Nolte, Chem. Rev., 2005, 105, 1445-1489.

11 M. A. C. Stuart, W. T. S. Huck, J. Genzer, M. Müller, C. Ober, M. Stamm, G. B. Sukhorukov, I. Szleifer, V. V. Tsukruk, M. Urban, F. Winnik, S. Zauscher, I. Luzinov and S. Minko, Nat. Mater., 2010, 9, 101-113.

12 C. G. Palivan, R. Goers, A. Najer, X. Zhang, A. Car and W. Meier, Chem. Soc. Rev., 2016, 45, 377-411.

13 L. Jiang, X. Huang, D. Chen, H. Yan, X. Li and X. Du, Angew. Chem., Int. Ed., 2017, 56, 2655-2659.

14 W. Zhang and C. Gao, J. Mater. Chem. A, 2017, 5, 16059-16104.

15 Z.-Q. Cao, Y.-C. Wang, A.-H. Zou, G. London, Q. Zhang, C. Gao and D.-H. Qu, Chem. Commun., 2017, 53, 8683-8686.

16 M.-H. Li and P. Keller, Soft Matter, 2009, 5, 927-937. 
17 S. Mura, J. Nicolas and P. Couvreur, Nat. Mater., 2013, 12, 991-1003.

18 H. C. Kolb, M. G. Finn and K. B. Sharpless, Angew. Chem., Int. Ed., 2001, 40, 2004-2021.

19 V. V. Rostovtsev, L. G. Green, V. V. Fokin and K. B. Sharpless, Angew. Chem., Int. Ed., 2002, 41, 2596-2599.

20 J.-F. Lutz and Z. Zarafshani, Adv. Drug Delivery Rev., 2008, 60, 958-970.

21 D. S. Tyler, J. Vappiani, T. Cañeque, E. Y. N. Lam, A. Ward, O. Gilan, Y.-C. Chan, A. Hienzsch, A. Rutkowska, T. Werner, A. J. Wagner, D. Lugo, R. Gregory, C. R. Molina, N. Garton, C. R. Wellaway, S. Jackson, L. MacPherson, M. Figueiredo, S. Stolzenburg, C. C. Bell, C. House, S.-J. Dawson, E. D. Hawkins, G. Drewes, R. K. Prinjha, R. Rodriguez, P. Grandi and M. A. Dawson, Science, 2017, 356, 1397-1401.

22 J.-F. Lutz, Angew. Chem., Int. Ed., 2008, 47, 2182-2184.

23 C. J. Hawker and K. L. Wooley, Science, 2005, 309, 1200-1205.

24 H. C. Kolb and K. B. Sharpless, Drug Discovery Today, 2003, 8, 1128-1137.

25 C. Deraedt, N. Pinaud and D. Astruc, J. Am. Chem. Soc., 2014, 136, 12092-12098.

26 H. Struthers, T. L. Mindt and R. Schibli, Dalton Trans., 2010, 39, 675-696.

27 B. Schulze and U. S. Schubert, Chem. Soc. Rev., 2014, 43, 2522-2571.

28 Y. H. Lau, P. J. Rutledge, M. Watkinson and M. H. Todd, Chem. Soc. Rev., 2011, 40, 2848-2866.

29 J. E. Moses and A. D. Moorhouse, Chem. Soc. Rev., 2007, 36, 1249-1262.

30 A. Maisonial, P. Serafin, M. Traïkia, E. Debiton, V. Théry, D. J. Aitken, P. Lemoine, B. Viossat and A. Gautier, Eur. J. Inorg. Chem., 2008, 298-305.

31 M. R. Jones, E. Mathieu, C. Dyrager, S. Faissner, Z. Vaillancourt, K. J. Korshavn, M. H. Lim, A. Ramamoorthy, V. W. Yong, S. Tsutsui, P. K. Stys and T. Storr, Chem. Sci., 2017, 8, 5636-5643.
32 I. Kim, N.-E. Lee, Y.-J. Jeong, Y.-H. Chung, B.-K. Cho and E. Lee, Chem. Commun., 2014, 50, 14006-14009.

33 H. Irie, K. Kamiya, T. Shibanuma, S. Miura, D. A. Tryk, T. Yokoyama and K. Hashimoto, J. Phys. Chem. C, 2009, 113, 10761-10766.

34 T. J. Meyer, Pure Appl. Chem., 1986, 58, 1193-1206.

35 A. P. de Silva, T. S. Moody and G. D. Wright, Analyst, 2009, 134, 2385-2393.

36 Y.-C. Hsieh, J.-L. Chir, H.-H. Wu, P.-S. Chang and A.-T. Wu, Carbohydr. Res., 2009, 344, 2236-2239.

37 L. Qin, L. Zhang, Q. Jin, J. Zhang, B. Han and M. Liu, Angew. Chem., Int. Ed., 2013, 52, 7761-7765.

38 Y. Zhao, Y. Li, Y. Li, C. Huang, H. Liu, S.-W. Lai, C.-M. Che and D. Zhu, Org. Biomol. Chem., 2010, 8, 3923-3927.

39 V. K. Praveen, C. Ranjith, E. Bandini, A. Ajayaghosh and N. Armaroli, Chem. Soc. Rev., 2014, 43, 4222-4242.

40 N. K. Allampally, A. Florian, M. J. Mayoral, C. Rest, V. Stepanenko and G. Fernández, Chem. - Eur. J., 2014, 20, 10669-10678.

41 Y. Mai and A. Eisenberg, Chem. Soc. Rev., 2012, 41, 5969-5985.

42 R. J. Hickey, J. Koski, X. Meng, R. A. Riggleman, P. Zhang and S.-J. Park, ACS Nano, 2014, 8, 495-502.

43 M. R. Molla, P. Rangadurai, L. Antony, S. Swaminathan, J. J. de Pablo and S. Thayumanavan, Nat. Chem., 2018, 10, 659-666.

44 C. Wang, L. Lu, W. Ye, O. Zheng, B. Qiu, Z. Lin, L. Guo and G. Chen, Analyst, 2014, 139, 656-659.

45 K. Sivakumar, F. Xie, B. M. Cash, S. Long, H. N. Barnhill and Q. Wang, Org. Lett., 2004, 6, 4603-4606.

46 Y. Liu, T. Pauloehrl, S. I. Presolski, L. Albertazzi, A. R. A. Palmans and E. W. Meijer, J. Am. Chem. Soc., 2015, 137, 13096-13105.

47 C. Besanceney-Webler, H. Jiang, T. Zheng, L. Feng, D. S. del Amo, W. Wang, L. M. Klivansky, F. L. Marlow, Y. Liu and P. Wu, Angew. Chem., Int. Ed., 2011, 50, 8051-8056.

48 M. M. Hanczyc, S. M. Fujikawa and J. W. Szostak, Science, 2003, 302, 618-622. 